\title{
Reaction-based fluorogenic probes for selective detection of cysteinyl oxidation in living cells
}

\section{Renan Ferreira}

Scripps Research

\section{Ling Fu}

State Key Laboratory of Proteomics, Beijing Proteome Research Center, National Center for Protein

Sciences, Beijing Institute of Radiation Medicine, Beijing 102206

Jing Yang

National Center for Protein Sciences

Kate Carroll ( $\square$ kcarroll@scripps.edu )

Scripps Research https://orcid.org/0000-0002-7624-9617

\section{Article}

Keywords: sulfenic acid, redox modification, CysOx

Posted Date: October 19th, 2021

DOl: https://doi.org/10.21203/rs.3.rs-956877/v1

License: (c) (i) This work is licensed under a Creative Commons Attribution 4.0 International License. Read Full License

Version of Record: A version of this preprint was published at Nature Communications on September 21st, 2022. See the published version at https://doi.org/10.1038/s41467-022-33124-z. 
1 Reaction-based fluorogenic probes for selective detection of cysteinyl oxidation in living cells 2

3 Renan B. Ferreira ${ }^{1}$, Ling $\mathrm{Fu}^{2,3}$, Jing $\mathrm{Yang}^{2}$, and Kate S. Carroll ${ }^{1 *}$

$4 \quad{ }^{1}$ Department of Chemistry, Scripps Research, Jupiter, Florida 33458, United States

$5 \quad{ }^{2}$ State Key Laboratory of Proteomics, Beijing Proteome Research Center, National Center for Protein

6 Sciences • Beijing, Beijing Institute of Lifeomics, Beijing 102206, China

$7 \quad$ Innovation Institute of Medical School, Medical College, Qingdao University, Qingdao 266071, China

$8 \quad$ *e-mail: kcarroll@scripps.edu 
10 Measuring reactive oxygen, nitrogen and sulfur species in cells is established technology, but turn-on

11 fluorescence tools for detecting the products of their reaction with protein cysteines remain essentially

12 unknown. Toward this goal, here we describe fluorogenic probes for sulfenic acid, a redox modification

13 of protein cysteines inextricably linked to signaling and oxidative stress. The probes, called CysOx1 and

14 CysOx2, are reaction-based, exhibit excellent cell permeability, rapid reactivity, and high selectivity with

15 minimal cytotoxicity. We applied CysOx2 in a cell-based 96-well plate assay to determine whether kinase

16 inhibitors modulate protein S-sulfenylation as well as O-phosphorylation. Analysis of these data revealed

17 an unexpected positive association of $S$-sulfenylation and inhibition of select kinases within the TK, AGC,

18 and CMGC families including GSK3, a multitasking Ser/Thr kinase and emerging therapeutic target for

19 neurodegenerative and mood disorders. Chemoproteomic mapping of sulfenic acid-modified cysteines

20 in GSK3 inhibitor-treated cells shows that sites of S-oxidation localize to regulatory cysteines within key

21 components of antioxidant defense systems. Our studies with CysOx probes offer up new insights into

22 kinase-inhibitor dependent modulation of sulfenylome dynamics and should accelerate future efforts in

23 the modern era of translational redox medicine. 
26 Redox reactions and oxidative stress have been implied in the etiology of numerous diseases as well as

27 in the aging process ${ }^{1}$. The modern era in translational redox medicine seeks to identify types, sources, 28 metabolizers, and targets of oxidants in order to develop effective drugs and therapies for ROSopathies ${ }^{2}$.

29 In this context, turn-on fluorescence probes also referred to as fluorogenic probes that measure oxidative 30 stress in living cells have proven invaluable for redox-related biomedical research ${ }^{3,4}$. Analogous tools for 31 detecting the reaction products between biological oxidants and proteins are grossly underdeveloped, a point exemplified by the lack of fluorogenic probes for detecting protein cysteine (i.e., cysteinyl) oxidation.

33 The latter issue is especially striking since protein cysteines are the major target of oxidants originating 34 from both endogenous and exogenous sources ${ }^{5}$.

Sulfenic acid (Cys-SOH) is a central redox modification of protein cysteines and is inextricably linked to oxidant signaling and stress $^{6}$. Sulfenic acid is generated by oxidation of a thiolate by reactive oxygen species (ROS) such as hydrogen peroxide $\left(\mathrm{H}_{2} \mathrm{O}_{2}\right)$ produced during cellular signaling and metabolism or by hydrolysis of sulfenyl halides, and very polarized nitrosothiols and disulfides ${ }^{7}$. If stabilized by the protein microenvironment, the thiol-sulfenic acid pair can operate as a switch that is triggered by redox changes to regulate protein function, structure, and localization ${ }^{8-11}$. The electrophilic sulfur atom in sulfenic acid can also react with a protein or low-molecular-weight thiol to form a disulfide ${ }^{12,13}$ or, under conditions of excess oxidative stress, can be oxidized further to sulfinic and sulfonic acids ${ }^{14}$. In either

44 scenario, stabilized or as transient intermediate, sulfenic acids are key modifications in the domain of 45 biological redox regulation.

Selective chemical detection of sulfenic acid is predicated on the chemical nature of this moiety in which

48 the sulfur can behave as both a nucleophile and electrophile. The latter reactivity has been exploited by numerous groups to develop carbon-based nucleophilic probes for detecting sulfenic acid in proteins and 50 cells $^{15}$. The first fluorescent probe for sulfenic acid was reported in $2007^{16}$, consisting of fluorescein 
51 attached at the C-4 position on 1,3-cyclohexanedione (Supplementary Fig. 1a). The nucleophilic C-2

52 carbon reacts selectively with sulfenic acid, but its application has been limited by the absence of "turn-

53 on" fluorescence or fluorogenic response. In 2016, our group identified phenaline-1,3-dione as a

54 chemical scaffold having fluorogenic potential vis-à-vis its reaction with sulfenic acid ${ }^{17}$ that was

55 subsequently adapted in the design of a ratiometric fluorescent probe ${ }^{18}$ (Fig. 1a and Supplementary Fig.

56 1b). However, exceedingly slow reaction rates of less than $0.01 \mathrm{M}^{-1} \mathrm{~s}^{-1}$ and the absence of turn-on

57 fluorescence signal precluded any practical application.

59 To address the significant gap in chemical tools available that report on cysteinyl oxidation in real-time in 60 living cells, here, we describe fluorogenic probes for detecting sulfenic acid (Fig. 1b and Supplementary

61 Fig. 1c). The probes are reaction-based, exhibit excellent cell permeability, rapid reactivity, and high 62 selectivity with minimal cytotoxicity. The chemical tools have enabled live-cell imaging studies to detect 63 redox-dependent changes in cysteinyl oxidation. Adaptation to a 96-well plate format enabled high64 throughput analysis of sulfenic acid in cells and this platform was used to screen a curated inhibitor 65 library, targeting major families of the human kinome, as potential modulators of cysteinyl oxidation. 66 These studies identified a cohort of GSK3 kinase inhibitors that elicited significantly increased sulfenic 67 acid modifications that localized to the regulatory cysteines of proteins involved in the response to 68 oxidative stress. Collectively, these studies report on first-in-class tools for facile fluorogenic detection of 69 cysteinyl oxidation in a variety of formats with broad application in future studies of redox biology and 70 drug pharmacology.

\section{Results}

73 To create a fluorogenic probe for sulfenic acid, three key alterations of the phenaline-1,3-dione scaffold 74 were envisioned: (i) replacement of a carbonyl group with the more electron withdrawing sulfonyl group 75 to increase the rate of reaction; (ii) appendage of an amino group to create an electron "push-pull system" 76 system, and (iii) fluorination of the aromatic core to enhance absorption and fluorescence wavelengths 
77 (Fig. 1b). Optimized synthetic schemes for the resulting compounds is described under Synthetic 78 Procedures. In brief, 6-bromo-2H-naphtho[1,8-bc]thiophen-2-one was prepared from naphthalene-1-thiol 79 in three steps as previously reported. The thiophenone core was hydrolyzed and dimethylated to produce 80 both thioether and ester functional groups and the thioether was oxidized to a sulfone group with mCPBA.

81 The brominated sulfone intermediate was then used in amination reactions, followed by condensation in 82 presence of $\mathrm{NaH}$ to produce the $\mathrm{C}$-nucleophile center. Finally, a fluorination step provided the desired 83 compounds.

a<smiles>CCCCCCC</smiles>

Enol

Favored in aqueous solution

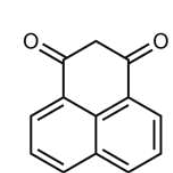

Keto

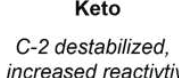
increased reactivtiy

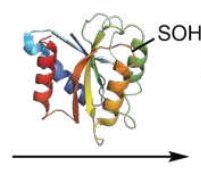

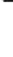

Protein Sulfenic acid
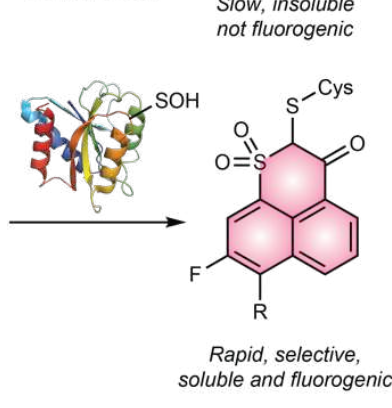

Rapid, selective, soluble and fluorogenic

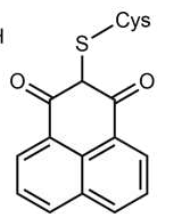

C-2 Sulfur adduct

Slow, insoluble

Cys
Fig. 1 | Design strategy for developing fluorogenic probes for detecting sulfenic acid. a, General structure of phenaline-1,3-dione scaffold and the tautomerization between enol and keto forms. Reaction between the nucleophilic C-2 and electrophilic sulfenic acid sulfur shifts the keto-enol equilibrium toward the keto form, which has fluorogenic potential, but is slow and poorly soluble. $\mathbf{b}$, Colored circles denote the position for introducing electron-withdrawing groups to favor fluorogenic reaction with sulfenic acid (left). The resulting fluorogenic reactionbased probes are rapid, selective, and water soluble. b $\begin{gathered}\text { Rate } \\ \text { acceleration }\end{gathered}$

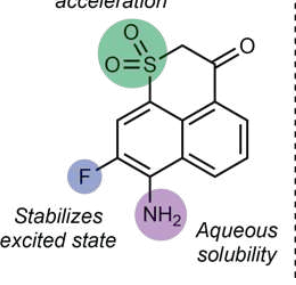

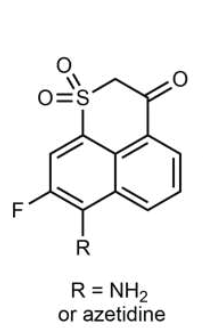

or azetidine

Kinetic and fluorescence characterization of phenaline-1,3-dione derivatives. We first sought to assess the reactivity of phenaline-1,3-dione (1) and its analogs with an established small-molecule model for cysteine sulfenic acid, known as $\mathrm{CSA}^{19,20}$ (Fig. 2a). Pseudo first-order rate constants were obtained for compound 1 and related analogs designed to evaluate features that modulate reactivity and fluorescence properties (Fig. 2b and Supplementary Fig. 2). Compound 1 exhibited modest kinetics $0.002 \mathrm{~s}^{-1}$ and fluorination of this scaffold at the C-2 position ( 2 and $\mathbf{3}$ ) decreased reactivity by more than two orders of magnitude. Replacement of one carbonyl group with a sulfonyl moiety (4) increased the reactivity of the phenaline-1,3-dione core by more than 1000-fold. C-2 fluorination (5) of the parent sulfonyl compound enhanced reactivity while introduction of a 7-amino group (6) decreased reaction rate. Although less reactive, the amino group enhanced the water solubility as compared to non-aminated 
96 group was replaced by the cyclic amine, azetidine (8) affording a compound with an observed reaction

97 rate of approximately $0.1 \mathrm{~s}^{-1}$. Overall, the sulfonyl group dramatically increased nucleophilic reactivity

98 towards the sulfenic acid electrophile, which was tempered somewhat by the installation of amino and 99 fluorine groups, as expected.

101 In subsequent experiments, we evaluated the fluorescence properties of sulfonyl derivatives 4 through 102 8. For this purpose, the reaction product between each analog and CSA was prepared and isolated from 103 milligram-scale reactions. Fluorescence spectra of the resulting adducts were then recorded in organic 104 solvent or aqueous solution and compared to that of non-adducted compounds alone (Fig. 2c-d, and 105 Supplementary Fig. 3). CSA adducts of $\mathbf{4}$ and $\mathbf{5}$ exhibited weak fluorescence signal and were essentially 106 nonfluorogenic. Furthermore, compounds 4 and $\mathbf{5}$ were only sparingly soluble in water. Compound 6 107 showed improved solubility but gave only weak fluorescence signal before and after reaction with CSA. 108 By contrast, CSA adducts of compounds 7 and 8 were fully soluble in PBS, exhibited bathochromic shifts 109 of excitation and emission maxima as well as increases in fluorescence intensity compared to $\mathbf{7}$ or $\mathbf{8}$ 110 alone, indicating that these compounds are fluorogenic for sulfenic acid (Fig. 2c-d). Specifically, the CSA1117 adduct gave a slight bathochromic shift of excitation and emission maxima of $3 \mathrm{~nm}$ with no change in 112 the Stokes shift. The bathochromic shift was more pronounced for the CSA-8 adduct (53 and $75 \mathrm{~nm}$ for 113 excitation and emission maxima, respectively) accompanied by an increase in the Stokes shift of $22 \mathrm{~nm}$. 114 The increase in fluorescence intensity was 2.3- for CSA-7 and 11.4-fold for CSA-8 (quantum yields 115 increased by 2.1- and 2.6-fold, respectively) compared to 7 and 8 alone. UV-Vis spectroscopy analysis 116 of 7 and 8 with their respective CSA adducts showed an increase in extinction coefficient of 1.7- and 6.4117 fold, respectively (Supplementary Table 1 and Supplementary Fig. 4-5). Finally, a bathochromic shift in 118 the absorption maxima of CSA-8 was also observed, in agreement with the shift observed in the 119 fluorescence spectra. Comparison of data obtained for compounds $\mathbf{7}$ and $\mathbf{8}$ to compound $\mathbf{6}$ demonstrates 120 that fluorination is essential for their fluorogenic response. 
a

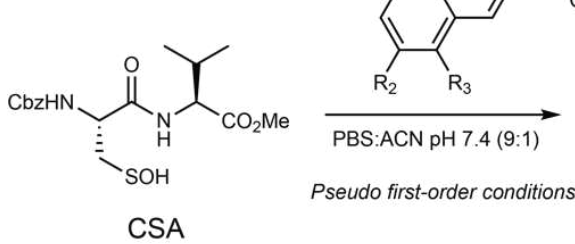

b

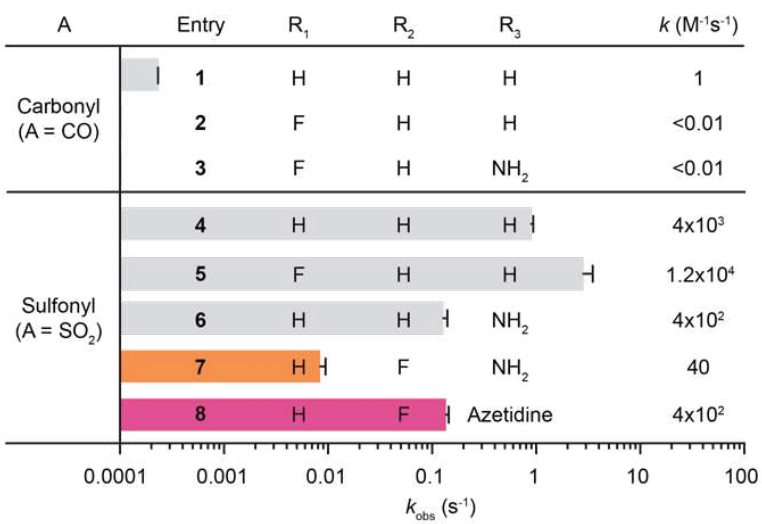

C

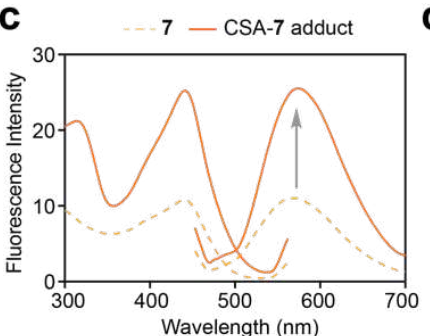

d

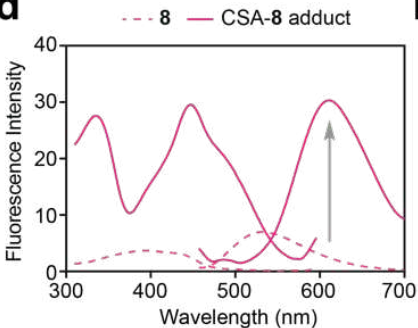

e
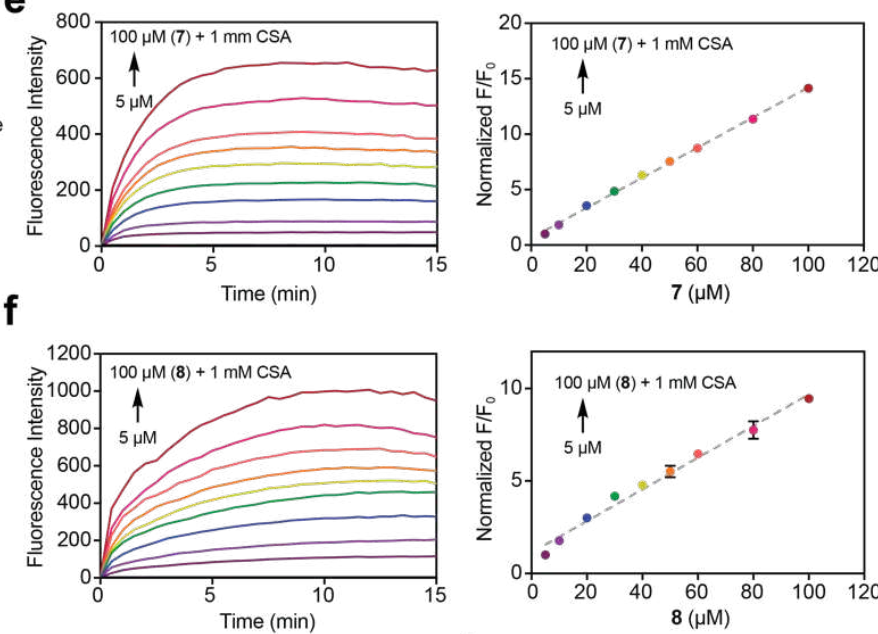

g
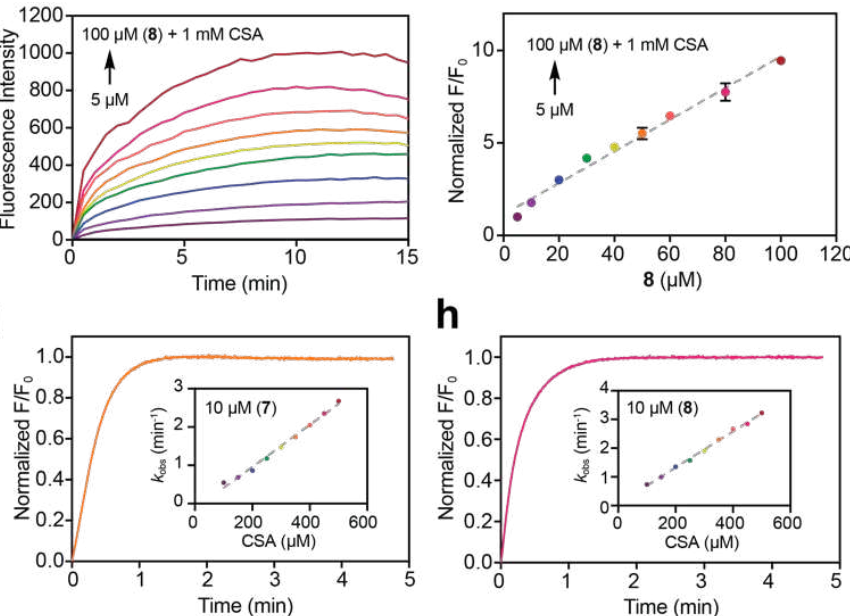

h
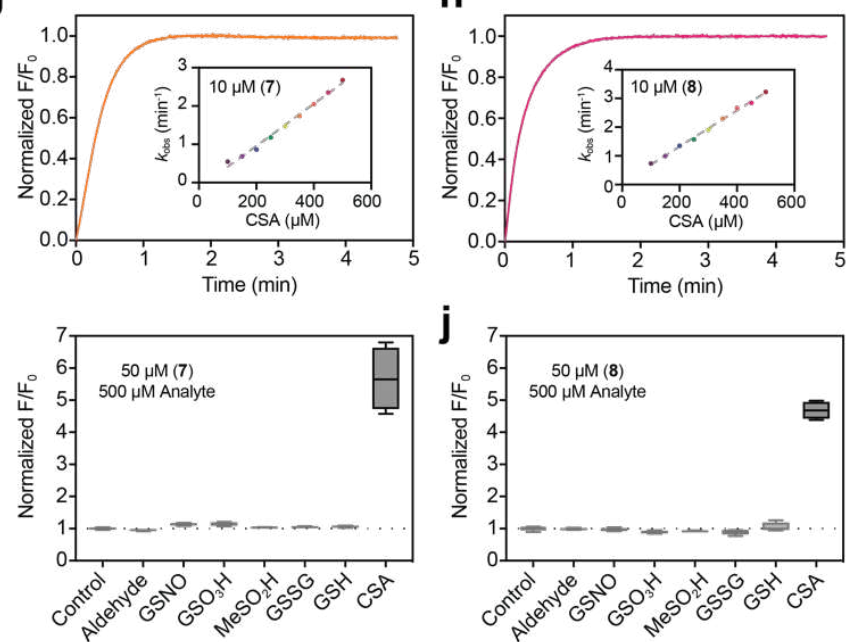

Fig. 2 | Chemical modification of the phenaline-1,3-dione scaffold yields fluorogenic probes for detecting sulfenic acid. a, Rate constants were measured under pseudo-first-order conditions with small-molecule sulfenic acid model, CSA, in excess over phenaline1,3-dione nucleophiles as described in Supplementary Methods. $\mathbf{b}, k_{\text {obs }}\left(s^{-1}\right)$ for compounds 1 through 8 were determined by curve fitting to a single exponential function. Second-order rate constant, $k$ is extrapolated from $k_{\text {obs }}$ divided by the concentration of the limiting agent. c, Absorption and emission spectra of 7 and isolated CSA-7 (250 $\mu \mathrm{M})$ in PBS:ACN pH 7.4 (9:1). d, Absorption and emission spectra of 8 and isolated CSA-8 $(250 \mu \mathrm{M})$ in PBS:ACN pH 7.4 (9:1). e, Fluorescence intensity monitored over time for the reaction between 7 (5 to $100 \mu \mathrm{M})$ and CSA (1 mM) after excitation at $443 \mathrm{~nm}$ (left). Fluorescence intensity at 15 min plotted versus the concentration of 7 (right). $\mathrm{n}$ = 3. f, Fluorescence intensity monitored over time for the reaction between 8 (5 to $100 \mu \mathrm{M})$ and CSA (1 mM) after excitation at $447 \mathrm{~nm}$ (left). Fluorescence intensity at 15 min plotted versus the concentration of $\mathbf{8}$ (right); $\mathbf{n}=3$. $\mathbf{g}$-h, Rate constants were measured under pseudo-first-order conditions with CSA in excess over phenaline-1,3-dione nucleophiles as described in Supplementary Methods. A representative trace from these collective reactions is shown. Linear fit of $k_{\text {obs }}\left(\mathrm{min}^{-1}\right)$ plotted versus CSA concentration gives $91 \pm 3$ and $105 \pm 2 \mathrm{M}^{-1} \mathrm{~s}^{-1}$ for 7 and $\mathbf{8}$, respectively (inset). $\mathrm{n}=3$. i-j, Box and whisker plot of normalized fluorescence intensity (F/F 0 ) for reaction between 7 or $8(50 \mu \mathrm{M})$ and various analytes $(1 \mathrm{mM})$ after $1 \mathrm{~h} ; \mathrm{n}=4$.

Next, we measured the fluorescence intensity of $\mathbf{7}$ and $\mathbf{8}$ in real-time reactions with CSA. Reactions 
$91 \pm 3$ and $105 \pm 2 \mathrm{M}^{-1} \mathrm{~s}^{-1}$ for 7 and $\mathbf{8}$, respectively (Fig. 2g,h). To screen for potential side reactions, we

127 evaluated the reactivity of $\mathbf{7}$ and $\mathbf{8}$ with a panel of potentially reactive biomolecules, such as aldehyde and disulfide electrophiles as well as related sulfur species including reduced glutathione, glutathione nitrosothiol, glutathione sulfinic and sulfonic acid. No significant reaction took place between $\mathbf{7}$ or 8 and any of the aforementioned compounds (Fig. i,j). Taken together, these studies indicate that compounds

$\mathbf{7}$ and 8, referred to hereafter as CysOx1 and CysOx2, exhibit reaction-based turn-on fluorescence, rapid reactivity, and high selectivity when evaluated in a small-molecule model of cysteine sulfenic acid.

Evaluation of CysOx reactivity and fluorescence in complex biological settings. Encouraged by our success in the CSA model, we then moved on to more targets with greater biological relevance and complexity. Toward this end, we examined CysOx probe reactivity in $\mathrm{C} 64,82 \mathrm{~S}$ glutathione peroxidase 3 (Gpx3; Fig. 3). This Gpx3 variant has one redox-sensitive cysteine, C36 that is readily oxidized to sulfenic acid using stoichiometric amounts of hydrogen peroxide $\left(\mathrm{H}_{2} \mathrm{O}_{2}\right)$ and has been well-validated as a model for the study of protein sulfenic acid reactivity ${ }^{19,20}$. Intact mass spectrometry (MS) analysis demonstrated that Gpx3 C36 sulfenic acid (Gpx3-SOH) formed the anticipated adduct with CysOx probes in high yield, while reduced Gpx3 (Gpx3-SH) was not modified (Fig. 3b,c). Having verified the correct protein adducts by MS, we next evaluated the fluorescence spectra of isolated Gpx3-CysOx adducts compared to that of free CysOx probes. Satisfyingly, CysOx1 and Cys Ox2 exhibited a "turn-on" fluorescence response after respective excitation at $357 \mathrm{~nm}$ and $394 \mathrm{~nm}$ (Fig. 3d,e). Of the two probes, Cys Ox2 showed a larger fluorescence enhancement of up to 4-fold. Fluorescence spectra of analogous real-time or in-situ reactions gave similar results and also demonstrate that fluorogenic response is only observed when all 147 three components - CysOx, $\mathrm{H}_{2} \mathrm{O}_{2}$, and $\mathrm{Gpx} 3$ - are present in the reaction (Fig. 3f,g). Compared to data obtained with CSA, the fluorescence spectra of Gpx3-CysOx adducts were blue-shifted by about $50 \mathrm{~nm}$.

149 Finally, the reaction products of CysOx probes and Gpx3-SOH were visualized by in-gel fluorescence 150 (Fig. 3h,i). Intense signal was detected in CysOx-treated Gpx3-SOH but not in Gpx3-SH, consistent with 151 the findings in our intact MS analysis. 

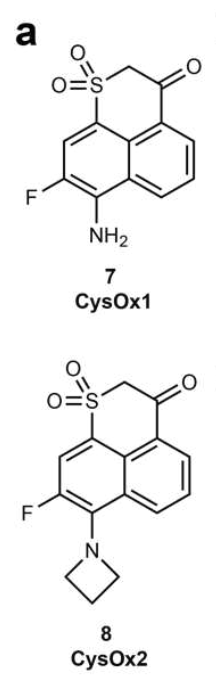

b

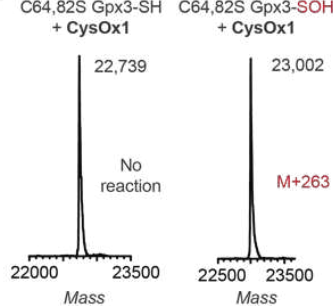

C64.82S

C64,82S Gp 3 -SOH + CysOx2
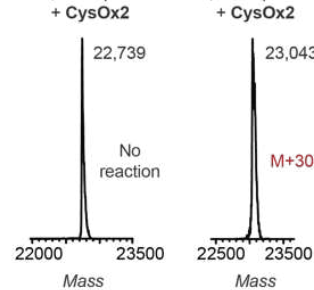

d

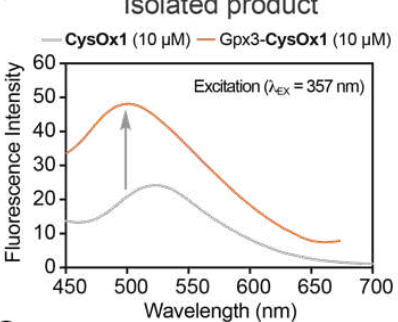

e

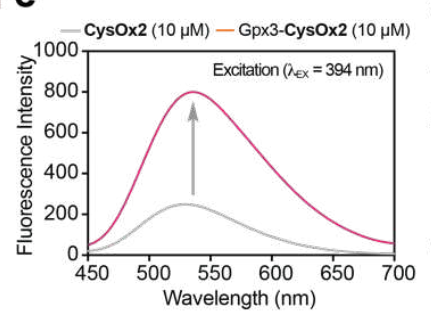

f

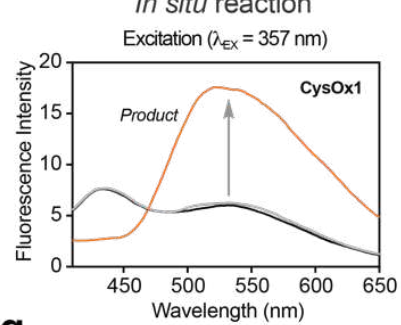

g

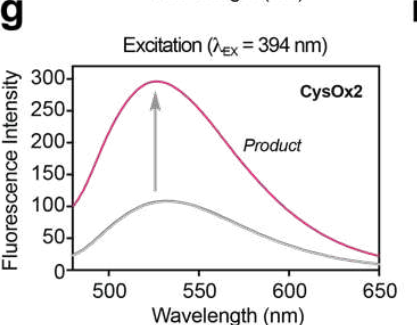

h

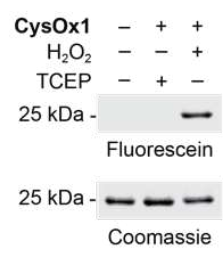

i

CysOx2 - + +

$\mathrm{H}_{2} \mathrm{O}_{2}--+$

TCEP - + -

$25 \mathrm{kDa}$ -

Fluorescein

$25 \mathrm{kDa}-$ - - -

Coomassie

Fig. 3 | CysOx1 and CysOx2 are reaction-based fluorogenic probes for protein cysteine (cysteinyl) sulfenic acid. a, Structures of CysOx1 and CysOx2. b-c Intact MS analysis of the reaction products of reduced or sulfenic acid forms of C64,82S Gpx3 (10 $\mu \mathrm{M})$ with CysOx1 or CysOx2 (1 mM) after $1 \mathrm{~h}$ in $50 \mathrm{mM}$ HEPES pH 7.4. d-e, Emission spectra of CysOx1 or CysOx2 alone (10 $\mu \mathrm{M})$ compared to purified Gpx3-CysOx1 or Gpx3-CysOx2 (10 $\mu \mathrm{M})$. Reactions with CysOx1 or CysOx2 were excited at $357 \mathrm{~nm}$ and $394 \mathrm{~nm}$, respectively. Spectra were recorded in $50 \mathrm{mM}$ HEPES pH 7.4. f-g, Emission spectra of CysOx1 or CysOx2 alone (10 $\mu \mathrm{M})$ or in combination with $\mathrm{H}_{2} \mathrm{O}_{2}$ $(15 \mu \mathrm{M})$ or $\mathrm{Gpx} 3(10 \mu \mathrm{M})$ or $\mathrm{H}_{2} \mathrm{O}_{2}(15 \mu \mathrm{M})$ and $\mathrm{Gpx} 3(10 \mu \mathrm{M})$. Only the reaction containing all three components gives a fluorogenic product. Reactions with CysOx1 or CysOx2 were excited at $357 \mathrm{~nm}$ and $394 \mathrm{~nm}$, respectively. Spectra were recorded in $50 \mathrm{mM} \mathrm{HEPES}$ $\mathrm{pH}$ 7.4. $\mathbf{~ h - i , ~ I n - g e l ~ f l u o r e s c e n c e ~ a n a l y s i s ~ o f ~ r e a c t i o n ~ p r o d u c t s ~ b e t w e e n ~} \mathrm{Gpx} 3(10 \mu \mathrm{M})$ and $\mathbf{C y s O x} \mathbf{1}$ or $\mathbf{C y s O} \mathbf{2} \mathbf{2}(1 \mathrm{mM})$ with or without $\mathrm{H}_{2} \mathrm{O}_{2}$ $(15 \mu \mathrm{M})$ or TCEP $(1 \mathrm{mM})$ after $1 \mathrm{~h}$ in $50 \mathrm{mM}$ HEPES $\mathrm{pH}$ 7.4.

Having established that CysOx probes are fluorogenic and selective in small-molecule and protein models of sulfenic acid, we next assessed their ability to enter live cells and provide a fluorogenic readout of endogenous S-sulfenylated proteins. For these experiments we employed glucose oxidase (GOX) at $0.2,2$, and $20 \mathrm{U} / \mathrm{mL}$ to provide continuous production of $\mathrm{H}_{2} \mathrm{O}_{2}$ in glucose-containing cell culture media ${ }^{21}$. HeLa cells incubated with CysOx1 or CysOx2 showed significant time- and GOX-dependent increases 
167 represents a viable strategy for fluorogenic detection of cysteinyl oxidation in the test tube and in real168 time in living cells.
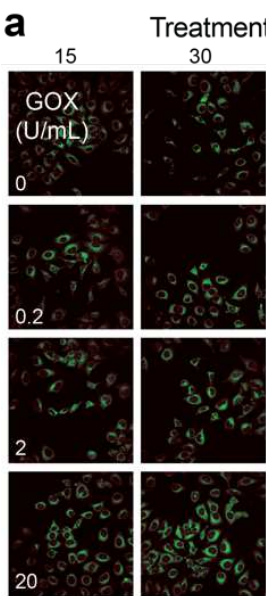
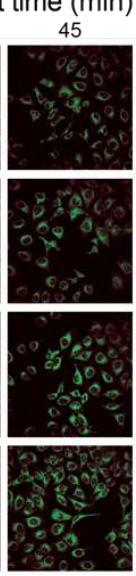
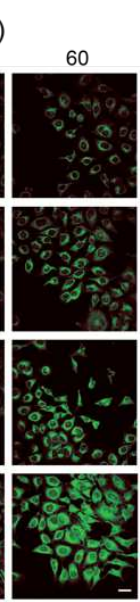

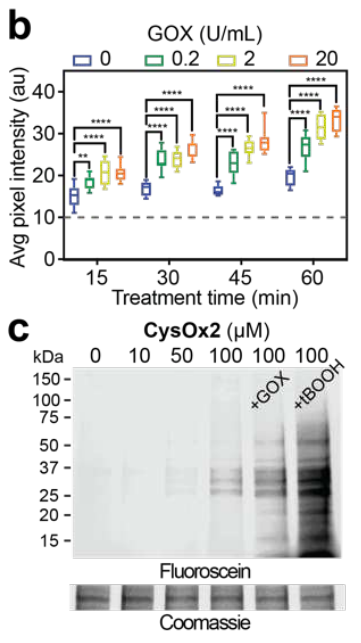

Fig. 4 | Cell permeable CysOx probes for no-wash live-cell microscopy of cysteine sulfenic acid in cells. a, No-wash live-cell confocal images of HeLa cells at different time points after the addition of CysOx2 $(10 \mu \mathrm{M})$ and GOX at the indicated concentrations $(0-20 \mathrm{U} / \mathrm{mL})$. $\lambda_{\mathrm{ex}}=458 \mathrm{~nm}$; scale bar $=50 \mu \mathrm{M}$. b, Box and whisker plot of the average pixel intensities from panel a. Data are representative of ten independent readings from five different frames. Error bars are \pm SEM. Variance was analyzed by two-way ANOVA test. ns $\mathrm{P}>0.05,{ }^{*} \mathrm{P}<0.05$, ** $\mathrm{P}<0.01,{ }^{* * *} \mathrm{P}<0.001,{ }^{* * *} \mathrm{P}<0.0001$ when compared against cells treated with probe only. c, In-gel fluorescence analysis of lysates derived from HeLa cells incubated with CysOx2 and GOX $(20 \mathrm{U} / \mathrm{mL})$ or $t-\mathrm{BOOH}(500 \mu \mathrm{M})$.

Application of CysOx2 to identify kinase inhibitors that modulate cysteinyl oxidation in cells.

171 Crosstalk between cysteine oxidation and other post-translational modifications such as phosphorylation

172 had been reported ${ }^{22,23}$ but the interplay between kinase inhibition and cysteinyl oxidation remains unclear.

173 To further showcase the utility of fluorogenic probes in the detection of cysteinyl oxidation and address

174 the question above, we subsequently adapted CysOx2 for use in a cell-based 96-well plate assay. After 175 probe concentration and treatment times were optimized for this scale (Supplementary Fig. 10) we 176 screened a curated library of kinase inhibitors was in HeLa cells. Analysis of the resulting data revealed 177 a 3-fold or greater increase in fluorescence for $8 \%$ (12 out of 154 compounds) of kinase inhibitors as 178 compared to vehicle-treated cells (Fig. 4a and Supplementary Table 2). Protein S-sulfenylation was 179 modulated by inhibition of RTKs as well as select kinases from the AGC and CMGC families, including 180 the multitasking Ser/Thr kinase GSK3 (Fig. 4b). Upon closer inspection, three of the ten compounds 181 associated with the greatest increase in fluorescence intensity (SB-216763, BIM-IX, and BIO) were 182 identified as GSK3 inhibitors (Fig. 4a-c), suggesting a relationship between this idiosyncratic kinase and 183 protein S-sulfenylation. Underscoring this point, application of the most effective inhibitor identified in this 184 screen, SB-216763 promoted a fluorescence increase equivalent to that of exogenously applied peroxide 
fluorescence imaging microscopy (Fig. e,f) and in-gel fluorescence analysis (Supplementary Fig. 11).

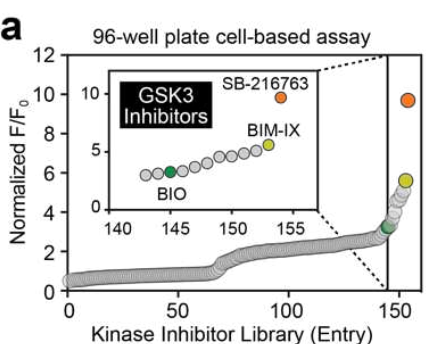

d

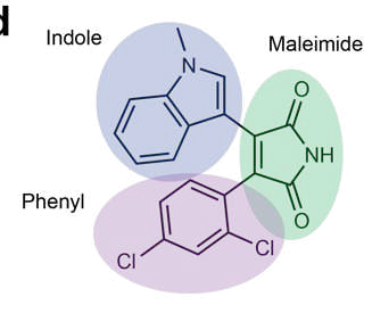

b

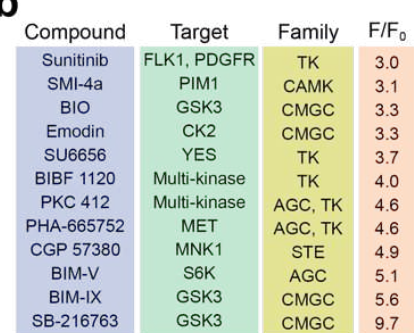

C

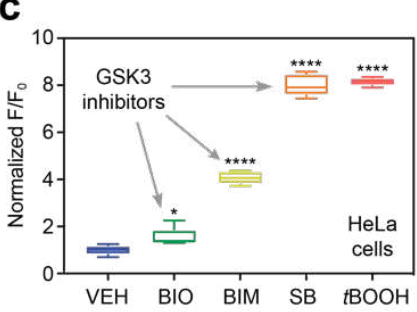

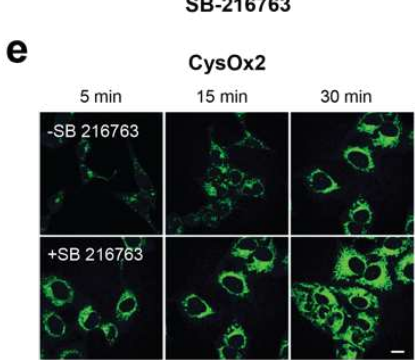

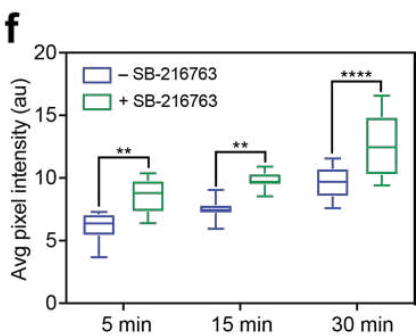

Fig. 5 | Multi-well based screening identifies kinase inhibitors that elevate cysteinyl oxidation in cells. a, Relative fluorescence intensity of HeLa cells treated with various kinase inhibitors $(10 \mu \mathrm{M})$ and CysOx2 (50 $\mu \mathrm{M})$ after $1 \mathrm{~h}$ at $37^{\circ} \mathrm{C}$. b, Twelve compounds demonstrated a relative increase in fluorescence intensity of 3-fold or greater. The primary target and kinase family for each compound is listed. c, Three GSK3 inhibitors were identified among the top ten hits: BIO, BIM-IV (BIM) and SB-216763 (SB). These inhibitors were purchased, their purity verified by LC-MS, and rescreened in HeLa cells at $1 \mu \mathrm{M}$ in the presence or absence of CysOx2 (50 $\mu \mathrm{M})$. The concentration of $t$-BOOH control was $2 \mathrm{mM}$. d, General structure of disubstituted maleimide-based GSK3 inhibitors. e, No-wash live-cell confocal images of HeLa cells at different time points after the addition of CysOx2 (5 $\mu \mathrm{M})$ in the presence or absence of SB$216763(0.1 \mu \mathrm{M})$. $\lambda_{\mathrm{ex}}=458 \mathrm{~nm}$; scale bar $=50 \mu \mathrm{M}$. f, Box and whisker plot of the average pixel intensities from panel e. Data are representative of ten independent readings from five different frames. Error bars are \pm SEM. Variance was analyzed by two-way ANOVA test. ns $\mathrm{P}>0.05,{ }^{*} \mathrm{P}<0.05,{ }^{* *} \mathrm{P}<0.01,{ }^{* * *} \mathrm{P}<0.001,{ }^{* * * *}$ $P<0.0001$ when compared against cells treated with probe only.

\section{Site-specific proteomic ID of cysteines that undergo S-sulfenylation in GSK3 inhibitor-treated}

cells. Having identified an interesting connection between GSK inhibition and cysteinyl oxidation, we

next sought to site-specifically and quantitatively profile dynamic changes in the HeLa S-sulfenylome

induced by GSK-3 inhibitors using BTD-based chemoproteomics (Fig. 5a). BTD is a clickable chemical probe for sulfenic acid detection ${ }^{24}$. In agreement with fluorescence screening data, SB-216763 treatment resulted in the most significant perturbation to the S-sulfenylome, with $56.3 \%$ measured S-sulfenylation events exhibiting at least a 1.5-fold change (Fig. 5b). Moreover, S-sulfenylation levels in nearly half of quantified sites with Uniprot functional annotations were changed by SB-216763 (Fig. 5c), implying broad regulation. Gene ontology classification further revealed that the sub-sulfenylome perturbed by GSK3 inhibition $\left(R_{\mathrm{L} / H}{ }^{S B-216763} \geq 1.5, R_{L / H}{ }^{B I M-I X} \geq 1.0, R_{L / H}{ }^{B i o} \geq 1.0\right)$ was enriched in many biological processes involved in antioxidant response, including regulatory cysteines of thioredoxin, thioredoxin reductase, glutaredoxin and glutaredoxin reductase (Fig. 5d,e). GSK3 inhibition also increased oxidation of PRDX6 C47 whose 
a
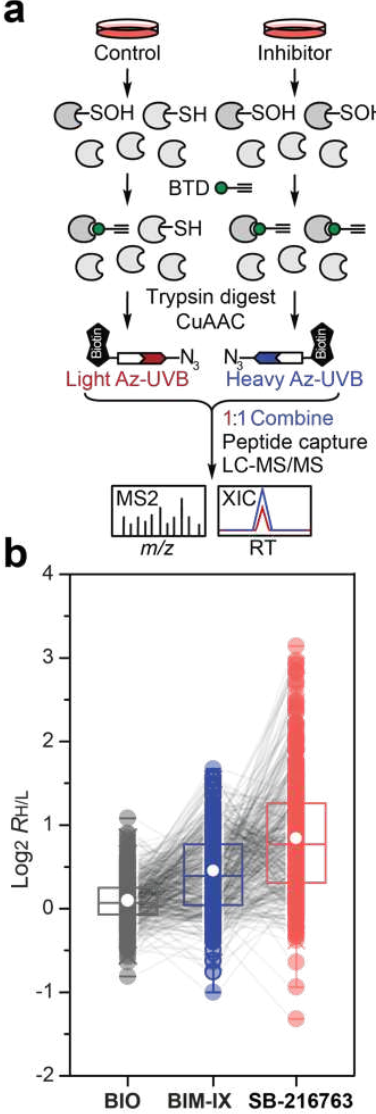

C
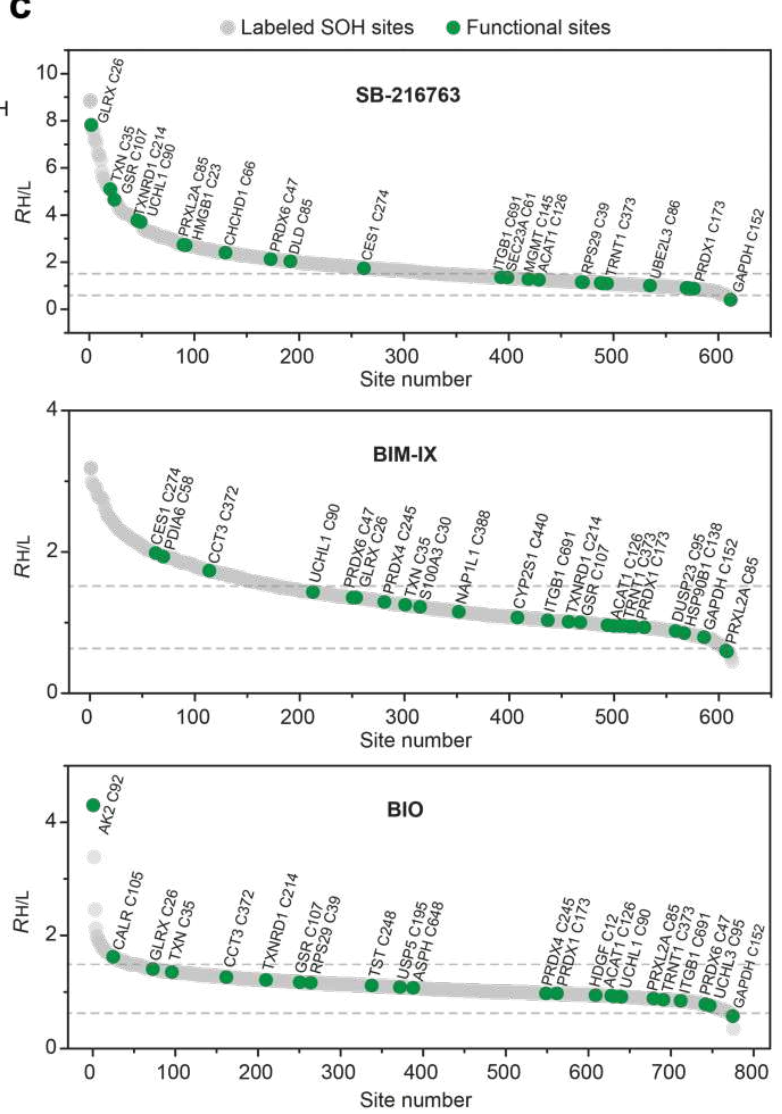

d

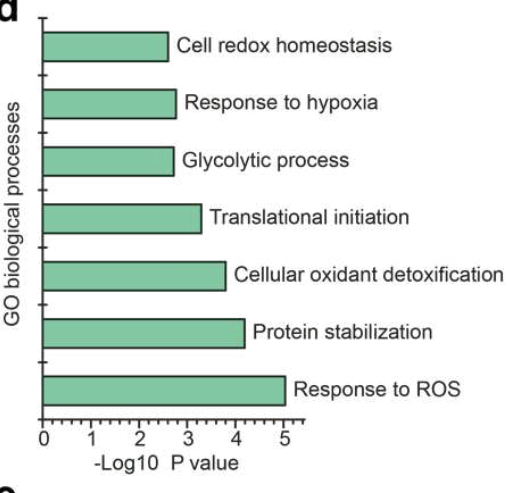

e

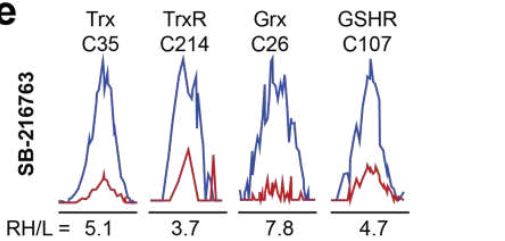

Fig. 6 | GSK3 inhibition selectively perturbs the S-sulfenylome. a, Workflow for the BTD-based quantitative S-sulfenylome analysis. HeLa cells were treated with vehicle or with GSK3 inhibitors as indicated, and then labeled with $5 \mathrm{mM}$ BTD in vitro. BTD-labeled proteomes with and without inhibitor treatment were digested into tryptic peptides, conjugated with heavy and light azido biotin reagents with a photocleavable linker, respectively. Light and heavy labeled samples then were combined, clicked, enriched with streptavidin beads. The photoreleased peptides were analyzed by LC-MS/MS for identification and quantitation. Heavy to light ratio calculated for each BTDlabeled cysteine residue provided a measure of its relative level in inhibitor-treated samples versus controls. $\mathbf{b}$, Box plot showing the sideby-side comparison of the quantitation results from three GSK3 inhibitors. c, Ranking plots showing the distribution of Log2 RH/L values for each GSK3 inhibitor. d, GO enrichment of the sub-sulfenylome perturbed by GSK3 inhibition. e, Representative extracted ion chromatograms (XICs) showing changes in BTD-labeled peptides from the proteins as indicated. The profiles for light- and heavy-labeled peptide are shown in red and blue, respectively. The average $R_{H / L}$ values calculated from two biological replicates are displayed below each individual XIC.

\section{Discussion}

In contrast to cysteinyl oxidation products, turn-on fluorescent probes for the detection of low-molecular- 
208 Such reagents have utility in cells and often show a degree of discrimination among different biothiols. 209 Despite such advances, the degree to which these tools report on cysteine residues in proteins is not 210 clear. Furthermore, this general class of reagents only detects biothiol oxidation as a loss of fluorescence 211 signal. To address this significant gap, this study presents the first instance of fluorogenic tools for direct 212 detection of protein cysteine or cysteinyl oxidation, specifically monitoring the formation of sulfenic acid.

214 The fluorogenic character of CysOx probes stems, at least in part, from the fluorescence quenching effect 215 of the phenaline-1,3-dione keto tautomer, in which nearly parallel carbonyl groups gives the largest large 216 dipole moment ${ }^{26}$. Reaction of the phenaline-1,3-dione scaffold at nucleophilic C-2 with the electrophilic 217 sulfur atom in sulfenic acid serves to stabilize the fluorogenic enol tautomer. A second key contributor to 218 the fluorescence of these probes is the electron-withdrawing fluorine atom attached to the naphthalene 219 core, which serves to stabilize the excited state ${ }^{27}$. A final feature of CysOx probes is the push-pull system 220 wherein the electron-donating amino group is in conjugation with fluorine via the intervening $\pi$-system ${ }^{28}$.

Another aspect of this study was to apply CysOx technology to identify kinase inhibitors that modulate Ssulfenylation in cells. The curated screening library used in this study includes inhibitors of lipid, receptor and non-receptor tyrosine, serine/threonine, and dual specificity kinases ${ }^{29}$. Several interesting patterns emerged from this screen. For instance, among inhibitors associated with a significant increase in cellular 226 S-sulfenylation, about $40 \%$ targeted kinases in the RTK family. This is consistent with reports from our 227 lab and others indicating that growth factor signaling is intimately linked to ROS metabolism in cells ${ }^{5,8}$. 228 The balance of "hits" within this cohort target members of the AGC and CMGC families and, of special 229 interest, three of these inhibit GSK3 function ${ }^{30}$. GSK3 in an unusual kinase in that it is constitutively 230 active and its substrates (over 100 are known) need to be pre-phosphorylated by another kinase, and it 231 is inhibited as opposed to activated by the two pathways known to converge on GSK3, the insulin and 232 Wnt pathways ${ }^{31}$. The elucidation of such patterns highlights an important strength of CysOx probes for 
233 identifying agents or conditions that modulate protein S-sulfenylation, an area of central importance to 234 many fields including covalent targeting of semi-conserved cysteines which is a growing strategy in drug $235 \operatorname{design}^{32,33}$.

237 To independently verify the relationship between GSK3 inhibition and cysteinyl oxidation we performed 238 chemoproteomic analysis using the sulfenic acid-selective probe, BTD. We found that GSK3 inhibitors 239 did indeed increase cellular S-sulfenylation with SB-216763 exhibiting the greatest effect, followed by 240 BIM-IX and BIO, consistent with the findings from our kinase inhibitor screen. In this regard, we note 241 that GSK3 inhibition activates Nrf2 for the transcription of antioxidant machinery ${ }^{34}$ and recent studies 242 suggest that the Nrf2 pathway can also be triggered by inactivation of thioredoxin and glutathione243 glutaredoxin systems, which are key reducing mechanisms in eukaryotes ${ }^{35}$. Consistent with this idea, S244 sulfenylation of regulatory redox-active cysteines in thioredoxin, thioredoxin reductase, glutathione 245 reductase and glutaredoxin, all increased with GSK3 inhibition and may represent a KEAP1-independent 246 means to activate Nrf2. Future research will be required to dissect this intriguing link in greater molecular 247 detail.

249 As with all technologies, it is worth noting current limitations. At present, the CysOx fluorophore presents 250 low brightness and quantum yields when compared to other dyes, such as fluorescein and BODIPY. This 251 is reflected in the low quantum yields (0.9-2.4\%) observed for the reaction product of sulfenic acid and 252 CysOx probes. This limitation does not hinder application of CysOx probes in the studies presented in 253 this work. However, attempts to further miniaturize the cell-based assay to a 384-well plate using Cys Ox2 254 failed, which may be addressed in future iterations of these tools with brighter probes and enhanced 255 dynamic range.

257 In summary, we have introduced a general chemical strategy for reaction-based fluorogenic detection of 258 cysteinyl sulfenic acid. Using this strategy we created two chemical probes, CysOx1 and CysOx2 that 
259 have excellent cell permeability, rapid reactivity, and high selectivity with minimal cytotoxicity. The kinetic 260 and spectroscopic properties of CysOx probes make them powerful first-in-class tools for live-cell 261 fluorescence imaging studies of redox-regulated biology in physiology and disease.

\section{Methods}

General Spectroscopy. Fluorescence spectra for studies not in cell culture were recorded on an Agilent Technologies Cary Eclipse Fluorescence Spectrophotometer running the Cary Eclipse v1.2 software. Absorbance spectra were recorded on a Varian Cary 300 Bio UV-Visible Spectrophotometer using Cary WinUV v4.20 software. Stock solutions of chemical probes were prepared in DMSO and then diluted in PBS (pH 7.4) at the indicated concentrations.

Preparation of probe-CSA adducts. To a solution of dipeptide cyclic sulfonamide CSA ( $36.6 \mathrm{mg}, 0.100$

$271 \mathrm{mmol})$ in $\mathrm{DCM}(3.0 \mathrm{~mL})$ was added a C-nucleophile probe $\left(0.100 \mathrm{mmol}, 1\right.$ equiv), followed by $\mathrm{Et}_{3} \mathrm{~N}(13.9$ $272 \mu \mathrm{L}, 0.100 \mathrm{mmol}, 1$ equiv). The reaction mixture was stirred at rt overnight. LC-MS analysis of the reaction 273 mixture indicated complete consumption of the starting materials and the formation of the desired CSA274 probe adduct as a single product. The reaction mixture was neutralized with TFA, diluted in DCM (20 $275 \mathrm{~mL})$, and washed with water $(20 \mathrm{~mL})$ and brine $(20 \mathrm{~mL})$. The organic phase was dried over $\mathrm{MgSO}_{4}$, 276 filtered and concentrated. The obtained products were purified via column chromatography $\left(\mathrm{SiO}_{2}, 0-\right.$ $277100 \%$ EtOAc in hexanes) and used as is in subsequent experiments.

C64,82S Gpx3 labeling with CysOx1 or CysOx2. C64,82S Gpx3-SH was spin-filtered into labeling 280 buffer (50 mM HEPES, $100 \mathrm{mM} \mathrm{NaCl}, \mathrm{pH}=7.4)$ using pre-equilibrated P30 columns (BioRad). The 281 concentration of C64,82S Gpx3-SH was determined by measuring the absorptivity at $280 \mathrm{~nm}(\varepsilon=24,410$ 282 $\left.\mathrm{M}^{-1} \mathrm{~cm}^{-1}\right)$. Solutions of $\mathrm{C} 64,82 \mathrm{~S} \mathrm{Gpx3}-\mathrm{SH}(20 \mu \mathrm{M})$ and CysOx1 or CysOx2 $(20 \mu \mathrm{M})$ were prepared in 
283 labeling buffer and kept in ice. The Cary Eclipse software was set to Kinetics mode using the fluorescence 284 excitation and emission maxima observed for Gpx3-CysOx adducts (357/498 nm for Gpx3-CysOx1 and $285394 / 535 \mathrm{~nm}$ for Gpx3-CysOx2). C64,82S Gpx3-SH (50 $\mu \mathrm{L})$ and CyxOx probe $(50 \mu \mathrm{L})$ were mixed in a 286 cuvette. Following sample insertion into the fluorimeter, $\mathrm{H}_{2} \mathrm{O}_{2}(100 \mu \mathrm{L}$ of $30 \mu \mathrm{M}$ made up in labeling 287 buffer) was fluorescence measurements were acquired. Three reactions were analyzed per chemical 288 probe. For control reactions, labeling buffer was added instead of selected components of the reaction.

In-gel fluorescence analysis of CysOx-labeled proteins. For Gpx3: C64,82S Gpx3 incubated with CysOx1 or CysOx2 $(21 \mu \mathrm{L})$ were boiled for 5 min in reducing loading buffer $(7 \mu \mathrm{L})$. For HeLa lysates derived from CysOx-treated cells: Lysate (20 $\mu \mathrm{g}$ protein) was diluted to a final volume of $30 \mu \mathrm{L}$ and boiled

293 for 5 min in non-reducing loading buffer $(10 \mu \mathrm{L})$. SDS-PAGE precast $4-20 \%$ or $4-15 \%$ gels (BioRad) 294 were electrophoresed in Tris-Glycine-SDS running buffer at $80 \mathrm{~V}$ for $10 \mathrm{~min}$ and then at $120 \mathrm{~V}$ for $60 \mathrm{~min}$. 295 Gels were then washed in $\mathrm{H}_{2} \mathrm{O}$ and imaged using the ChemiDoc MP imaging system (BioRad). Settings 296 were as follows: Fluorescein (epi-blue 460-490 nm excitation and 577-613 nm emission filter). After 297 fluorescence imaging, gels were stained in Commassie Brilliant Blue solution for 5 min at rt, washed in 298 destaining solution $\left(\mathrm{H}_{2} \mathrm{O} / \mathrm{MeOH} / \mathrm{AcOH}\right.$ 5:4:1) overnight, and then scanned by ChemiDoc MP to visualize 299 Coomassie staining.

301 Fluorescence cell imaging of live cells treated with CysOx probes. HeLa cells (seeding population: $3028.0 \times 10^{4}$ cells/well) were incubated in EMEM (+10\% FBS) in 6-well glass bottom plates at $37^{\circ} \mathrm{C}$. At $70-$ $30380 \%$ confluence, media was aspirated, and cells were washed twice with DPBS and serum-free EMEM 304 was added to wells. After incubation for $16 \mathrm{~h}$ at $37^{\circ} \mathrm{C}$ media was aspirated, and cells were washed twice 305 with DPBS. For GOX treatment: Cells were treated with CysOx probe and GOX at the indicated 306 concentrations in DPBS containing $0.1 \%$ DMSO and glucose $(1 \mathrm{mg} / \mathrm{mL})$. At the indicated time points, 307 well were directly analyzed in an Olympus FluoView IX81 confocal microscope using the FV10-ASW 3.0 
software. For $\mathrm{tBOOH}$ treatment: Oxidant was added at the indicated concentrations in EMEM for 10 min at $37^{\circ} \mathrm{C}$ followed by the addition of CysOx probe at the indicated concentration for additional 15 min at $37^{\circ} \mathrm{C}$. The media was then aspirated, cells washed twice with DPBS, and fresh DPBS added. The wells were then analyzed in an Olympus FluoView IX81 confocal microscope using FV10-ASW 3.0 software. The $458 \mathrm{~nm}$ laser channel was used to excite incorporated CysOx probes. Fluorescence signal was

313 filtered using a SDM560 dichroic mirror, followed by BA505-605 band pass filter to observe the CysOx 314 fluorescence. Five frames were recorded per condition. Images were analyzed using ImageJ software, 315 where the pixel intensity of the cellular cytoplasmic regions was measured with at least 10 measurements 316 per condition.

Multi-well cell-based screening assay to identify kinase Inhibitors that modulate S-sulfenylation.

319 HeLa cells were incubated in EMEM supplemented with 10\% FBS in black 96-well plates with clear 320 bottoms (seeding population: $2 \times 10^{4}$ cells/well) at $37^{\circ} \mathrm{C}$. At $90 \%$ confluence $(48 \mathrm{~h})$, media was aspirated, 321 cells washed twice with PBS and serum-free EMEM was added to the wells. After incubation for $16 \mathrm{~h}$ at $32237^{\circ} \mathrm{C}$ media was aspirated, cells were washed twice with PBS, and then DPBS (90 $\left.\mu \mathrm{L}\right)$ was added to 323 each well. CysOx2 $(50 \mu \mathrm{M})$ and kinase inhibitor (10 $\mu \mathrm{M}$, Kinase Screening Library - Cayman Chemical, 324 item no. 10505) in DPBS containing 1\% DMSO was added each well for a total volume per well of 100 $325 \mu \mathrm{L}$. Cells were then incubated at $37^{\circ} \mathrm{C}$ for $60 \mathrm{~min}$. Selected control wells did not receive kinase inhibitor 326 and were instead treated with either PBS (negative control) or $t \mathrm{BOOH}(200 \mu \mathrm{M}$, positive control). 327 Following the incubation period, DPBS $(100 \mu \mathrm{L})$ and extracellular fluorescence quencher $(20 \mu \mathrm{L}$, Solution 328 C of Beta-Lactamase Loading Solutions Kit from Life Technologies, part number K1048) were added to 329 each well prior to measurement in a Molecular Devices SpectraMax M5 plate reader and analyzed using 330 SoftMax Pro v5.4 software. The fluorescence parameters of CysOx2 were selected according to the 331 maxima measured in the fluorescence spectra (excitation/emission: 447/606 nm). Preliminary screening 332 to identify hits was performed in duplicate and compounds exhibiting more than a 2.5-fold increase in 333 fluorescence were subsequently confirmed in quintuplicate according to the same procedure. 


\section{Reporting Summary}

336 Further information on research design is available in the Nature Research Reporting Summary linked to 337 this article.

\section{Data availability}

340 All data associated with this study are available in the published article and its Supplementary 341 Information.

References

344 1. Forman, H. J. \& Zhang, H. Targeting oxidative stress in disease: promise and limitations of 345 antioxidant therapy. Nat. Rev. Drug Discov. 20, 689-709 (2021).

$346 \quad 2 . \quad$ Ghezzi, P. \& Mooradian, A. D. Demystifying Oxidative Stress. in Reactive Oxygen Species. 347 Handbook of Experimental Pharmacology (eds. Schmidt, H. H. H. W., Ghezzi, P. \& Cuadrado, $348 \quad$ A.) 264, 3-26 (Springer, Cham, 2020).

349 3. Dickinson, B. C. \& Chang, C. J. Chemistry and biology of reactive oxygen species in signaling or 350 stress responses. Nat. Chem. Biol. 7, 504-511 (2011).

351 4. Chan, J., Dodani, S. C. \& Chang, C. J. Reaction-based small-molecule fluorescent probes for 352 chemoselective bioimaging. Nat. Chem. 4, 973-984 (2012).

353 5. Sies, H. \& Jones, D. P. Reactive oxygen species (ROS) as pleiotropic physiological signalling 354 agents. Nat. Rev. Mol. Cell Biol. 21, 363-383 (2020).

355 6. Paulsen, C. E. \& Carroll, K. S. Cysteine-mediated redox signaling: Chemistry, biology, and tools 
for discovery. Chem. Rev. 113, 4633-4679 (2013).

357 7. Gupta, V. \& Carroll, K. S. Sulfenic acid chemistry, detection and cellular lifetime. Biochim. 358 Biophys. Acta 1840, 847-875 (2014).

359 8. Paulsen, C. E. et al. Peroxide-dependent sulfenylation of the EGFR catalytic site enhances 360 kinase activity. Nat. Chem. Biol. 8, 57-64 (2011).

361 9. Heppner, D. E. et al. Direct cysteine sulfenylation drives activation of the Src kinase. Nat. 362 Commun. $2018919,1-11(2018)$.

363 10. Pei, J.-F. et al. Diurnal oscillations of endogenous $\mathrm{H}_{2} \mathrm{O}_{2}$ sustained by p66Shc regulate circadian 364 clocks. Nat. Cell Biol. 21, 1553-1564 (2019).

365 11. Meng, J. et al. Global profiling of distinct cysteine redox forms reveals wide-ranging redox 366 regulation in C. elegans. Nat. Commun. 12, 1-13 (2021).

12. Bersweiler, A. et al. A scaffold protein that chaperones a cysteine-sulfenic acid in $\mathrm{H}_{2} \mathrm{O}_{2}$ signaling. Nat. Chem. Biol. 13, 909-915 (2017).

13. Beedle, A. E. M., Lynham, S. \& Garcia-Manyes, S. Protein S-sulfenylation is a fleeting molecular switch that regulates non-enzymatic oxidative folding. Nat. Commun. 2016 71 7, 1-10 (2016).

371 14. Akter, S. et al. Chemical proteomics reveals new targets of cysteine sulfinic acid reductase. Nat. Chem. Biol. 14, 995-1004 (2018).

373 15. Shi, Y. \& Carroll, K. S. Activity-based sensing for site-specific proteomic analysis of cysteine oxidation. Acc. Chem. Res. 53, 20-31 (2019).

16. Poole, L. B. et al. Fluorescent and affinity-based tools to detect cysteine sulfenic acid formation in proteins. Bioconjug. Chem. 18, 2004-2017 (2007).

377 17. Gupta, V., Paritala, H. \& Carroll, K. S. Reactivity, selectivity, and stability in sulfenic acid detection: A comparative study of nucleophilic and electrophilic probes. Bioconjug. Chem. 27, 
18. Tom, C. T. M. B. et al. Chemoselective ratiometric imaging of protein S-sulfenylation. Chem. Commun. 53, 7385-7388 (2017).

19. Gupta, V. \& Carroll, K. S. Profiling the reactivity of cyclic C-nucleophiles towards electrophilic sulfur in cysteine sulfenic acid. Chem. Sci. 7, 400-415 (2015).

20. Shi, Y. \& Carroll, K. S. Parallel evaluation of nucleophilic and electrophilic chemical probes for sulfenic acid: Reactivity, selectivity and biocompatibility. Redox Biol. 46, 102072 (2021).

21. S, M., G, M. \& GN, W. The GOX/CAT system: a novel enzymatic method to independently control hydrogen peroxide and hypoxia in cell culture. Adv. Med. Sci. 54, 121-135 (2009).

22. Su, Z. et al. Global redox proteome and phosphoproteome analysis reveals redox switch in Akt. Nat. Commun. 2019101 10, 1-18 (2019).

23. Dansen, T. B. et al. Redox-sensitive cysteines bridge p300/CBP-mediated acetylation and FoxO4 activity. Nat. Chem. Biol. 5, 664-672 (2009).

24. Fu, L., Liu, K., Ferreira, R. B., Carroll, K. S. \& Yang, J. Proteome-wide analysis of cysteine Ssulfenylation using a benzothiazine-based probe. Curr. Protoc. Protein Sci. 95, e76 (2019).

25. Yue, Y., Huo, F. \& Yin, C. The chronological evolution of small organic molecular fluorescent probes for thiols. Chem. Sci. 12, 1220-1226 (2021).

26. Cook, G. \& Feltman, P. M. Determination of solvent effects on keto-enol equilibria of 1,3dicarbonyl compounds using NMR. J. Chem. Educ. 84, 1827-1829 (2007).

27. Casa, S. \& Henary, M. Synthesis and applications of selected fluorine-containing fluorophores. Molecules 26, 1160 (2021).

28. Meier, H. Conjugated oligomers with terminal donor-acceptor substitution. Angew. Chemie Int. Ed. 44, 2482-2506 (2005). 
402

403

404

405

406

407

408

409

410

411

412

413

414

415

416

417

418

419

420

421

422

423

424

425

29. Kinase Screening Library (96-Well) | Cayman Chemical. Available at:

https://www.caymanchem.com/product/10505/kinase-screening-library-(96-well). (Accessed: 20th September 2021)

30. Cohen, P. \& Goedert, M. GSK3 inhibitors: Development and therapeutic potential. Nat. Rev. Drug Discov. 3, 479-487 (2004).

31. Beurel, E., Grieco, S. F. \& Jope, R. S. Glycogen synthase kinase-3 (GSK3): Regulation, actions, and diseases. Pharmacol. Ther. 148, 114-131 (2015).

32. Singh, J., Petter, R. C., Baillie, T. A. \& Whitty, A. The resurgence of covalent drugs. Nat. Rev. Drug Discov. 10, 307-317 (2011).

33. Fu, L. et al. A quantitative thiol reactivity profiling platform to analyze redox and electrophile reactive cysteine proteomes. Nat. Protoc. 15, 2891-2919 (2020).

34. M, C. \& M, A. GSK-3ß, a double-edged sword in Nrf2 regulation: Implications for neurological dysfunction and disease. F1000Research 7, (2018).

35. Lu, J. \& Holmgren, A. The thioredoxin antioxidant system. Free Radic. Biol. Med. 66, 75-87 (2014).

\section{Acknowledgements}

We thank Dr. Louis Scampavia and Dr. Timothy Spicer (The Scripps Research Institute) for helpful discussions in high-throughput screening and providing the fluorescence quencher used in the cell-based microplate assay. We thank Professor Laura Bohn and Dr. Edward Stahl (The Scripps Research Institute) for training in fluorescence microscopy and providing technical support and maintenance to the microscope. We thank Alta Johnson (The Scripps Research Institute) for training in flow cytometry. We thank Longqin Sun and Tuo Zhang (Beijing Qinglian Biotech Co., Ltd) for their help and technical supports. The work was supported by grants from the Natural Science Foundation of China (21922702) 
426 and the State Key Laboratory of Proteomics (SKLP-K201703 and SKLP-K201804) to J.Y., and NIH 427 (GM102187 and CA222849) to K.S.C.

428

\section{Author Contributions}

430 R.B.F, K.S.C. and J.Y. designed the experiments, analyzed data and all authors wrote the 431 manuscript and provided input. R.B.F. synthesized probes, performed rate studies, compound 432 characterization, acquisition of UV-Vis and fluorescence spectra, fluorescence microscopy for 433 live-cell imaging, fluorescence microplate assays, intact MS, SDS-PAGE, and in-gel 434 fluorescence analysis of CysOx-Gpx3 adducts. J.Y. and L.F. performed chemoproteomic 435 experiments and analyzed the data.

436

437 Competing Interests statement

438 The authors declare no competing financial interests. 


\section{Supplementary Files}

This is a list of supplementary files associated with this preprint. Click to download.

- FerreiraetalSupplementaryInformation.pdf

- FerrieraetalSyntheticProcedures.pdf

- SupplementaryTable2.xlsx

- katescarrollrsflat.pdf 